\title{
Online Design Bug Detection: RTL Analysis, Flexible Mechanisms, and Evaluation
}

\author{
$\ddagger$ Advanced Computer Architecture Lab \\ University of Michigan \\ \{kypros, austin\}@umich.edu
}

Kypros Constantinides $\ddagger$

\begin{abstract}
Higher level of resource integration and the addition of new features in modern multi-processors put a significant pressure on their verification. Although a large amount of resources and time are devoted to the verification phase of modern processors, many design bugs escape the verification process and slip into processors operating in the field. These design bugs often lead to lower quality products, lower customer satisfaction, diminishing brand/company reputation, or even expensive product recalls.

This paper proposes a flexible, low-overhead mechanism to detect the occurrence of design bugs during on-line operation. First, we analyze the actual design bugs found and fixed in a commercial chipmultiprocessor, Sun's OpenSPARC T1, to understand the behavior and characteristics of design bugs. Our RTL analysis of design bugs shows that the number of signals that need to be monitored to detect design bugs is significantly larger than suggested by previous studies that analyzed design bugs at a higher level using processor errata sheets. Second, based on the insights obtained from our analyses, we propose a programmable, distributed online design bug detection mechanism that incorporates the monitoring of bugs into the flip-flops of the design. The key contribution of our mechanism is its ability to monitor all control signals in the design rather than a set of signals selected at design time. As a result, it is very flexible: when a bug is discovered after the processor is shipped, it can be detected by monitoring the set of control signals that trigger the design bug.

We develop an RTL prototype implementation of our mechanism on the OpenSPARC T1 chip multiprocessor. We found its area overhead to be $10 \%$ and its power consumption overhead to be $3.5 \%$ over the whole OpenSPARC T1 chip.
\end{abstract}

\section{Introduction}

The Challenges of Correct Design - The advent of chipmultiprocessing has led to unprecedented levels of chip integration. Today, most general purpose processor chips are equipped with multiple cores, multiple levels of coherent memory, on-chip interconnection networks, and memory and I/O controllers. At the same time, processors are augmented with new technologies such as virtualization, dynamic power management, and 64-bit extensions. Complex interactions between these modules, as well as the complexity of the modules themselves, put a tremendous pressure in the verification of the system. Although the verification phase of modern processors can consume a large portion of the design cycle [3], require significant amount of resources [7], and utilize state-of-the-art verification techniques, design bugs (also known as errata, design defects, or design errors) still slip into the final products and "buggy" processors find their way into the field.

This trend is clearly shown in Figure 1. We studied the errata documentation of five recent Intel processors and found that the rate of design bugs discovered after product release has more than doubled in the latest generation of processors. ${ }^{1}$ The graph shows the number

\footnotetext{
${ }^{1}$ The data is extracted from the processors' errata documentation $[12,11,8$, $10,9]$.
}

\author{
Onur Mutlu§ Todd Austin $\ddagger$ \\ $\S$ Microsoft Research and Carnegie Mellon University \\ onur@\{microsoft.com,cmu.edu $\}$
}

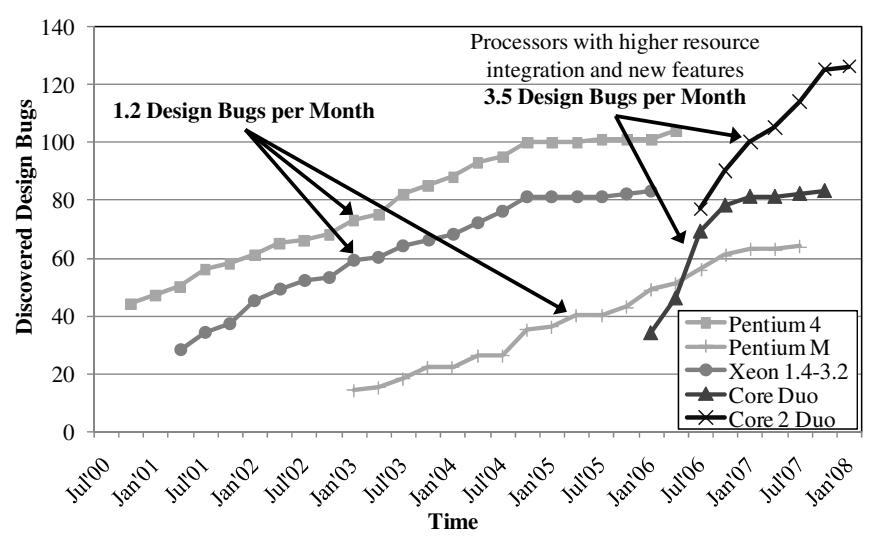

Figure 1. Timeline of discovered design bugs over the lifetime of five Intel processors

of discovered design bugs over the lifetime of five Intel processors. The Pentium 4, Pentium M, and the Xeon 1.4-3.2 processors exhibit a similar trend with an average of 1.2 design bugs discovered per month during their lifetime. ${ }^{2}$ On the other hand, the higher chip-level integration of resources and the addition of new features in the Core Duo and Core 2 Duo processors resulted in more design bugs. For example, although the Core Duo dual-core processor was derived from the Pentium M single-core processor and had the same architecture, it exhibited a much higher rate of design bugs than its predecessor. Specifically, the design bug discovery rate of the two multi-core processors is 3.5 design bugs per month, almost triple that of their single-core predecessors. This trend is expected to worsen in the future as technology scaling will allow for more diverse resources to be integrated into a single chip.

Why is Online Bug Detection Needed? Today, design bugs are treated with ad-hoc heuristic techniques that seek to avoid the occurrence of design bugs through software and hardware configuration changes [16]. A common approach employed by such techniques to avoid the occurrence of design bugs is disabling some processor features that trigger the design bugs (e.g., support for cache prefetching [16], dynamic power management [1], etc.). However, this often leads to reduced product quality/performance and lower customer satisfaction. Furthermore, when such workarounds are not possible, design bugs can lead to expensive product recalls [27] and a potentially diminishing brand/company reputation.

Augmenting a design with a mechanism that enables a systematic approach to detect and avoid design bugs after the product release and while the system is operating in the field can offer the following benefits:

1. Faster design cycle and time to market. Today, a significant fraction of the verification phase is spent to discover a very small number of design bugs [6]. This time can be saved by discovering and fixing that small number of design bugs in the field after product release.

\footnotetext{
${ }^{2}$ We suspect that the reason why the Pentium M processor had less design bugs than the other two processors is because it was based on the matured Intel P6 architecture.
} 


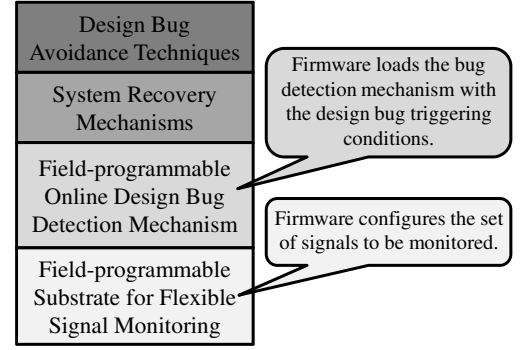

Figure 2. High-level overview of an in-the-field design bug detection and avoidance framework

2. Reduce the risk of expensive product recalls (and potentially damaged company reputation) due to ad-hoc heuristic techniques that might not be able to avoid a discovered design bug. A systematic online design bug detection technique increases the probability of successfully dealing with the design bug and avoiding expensive recalls.

3. Avoid potential impact to product quality and customer satisfaction due to the use of conventional techniques that disable design features to avoid design bugs. Instead, online design bug detection allows the system to operate with all its features enabled and recover the system only when the design bug occurs. Therefore, during bug-free execution the system is operating under its original specifications.

Online Design Bug Detection and Avoidance - Figure 2 provides a high-level overview of the in-the-field design bug detection and avoidance framework which we will describe in this paper. The framework has four layers: 1) The bottom layer that provides a fieldprogrammable substrate for flexible signal monitoring. This substrate is programmed by special firmware at system startup to select the set of signals that are required to be monitored for design bug detection. 2) A field-programmable design bug detection mechanism that checks if the monitored signals match with a bug triggering condition. The mechanism is programmed by special firmware at system startup with the bug triggering conditions. 3) A system recovery mechanism that rolls back the system state to the last correct state when a design bug occurrence is detected. 4) Design bug avoidance techniques that are activated after a design bug detection to guide execution around the bug triggering conditions and avert the design bug. In this paper we focus on the first two layers and provide a novel mechanism for performing flexible signal monitoring and online design bug detection.

\subsection{Contributions of this Work}

This work makes the following contributions in the area of online design bug detection:

- We further the understanding in online design bug detection by performing a rigorous analysis of the design bugs in the OpenSPARC T1 chip-multiprocessor. Unlike previous works that based their design bug analyses on high-level abstract descriptions of the processor errata documentation, our analysis is performed at the RTL model of the design, thus enabling the extraction of low-level information directly related to the actual hardware implementation. To the best of our knowledge this is the first RTL design bug analysis study published to date.

- Based on the insights obtained from our RTL analysis of design bugs, we propose a novel distributed online bug detection mechanism. Unlike the mechanisms proposed in previous work, our mechanism can concurrently monitor all the control signals in the design that can trigger a design bug.

- We provide a detailed hardware implementation of our mechanism. Unlike previously proposed mechanisms that route selected signals from the source flip-flops to a centralized monitoring mechanism that checks for bug triggering conditions, our

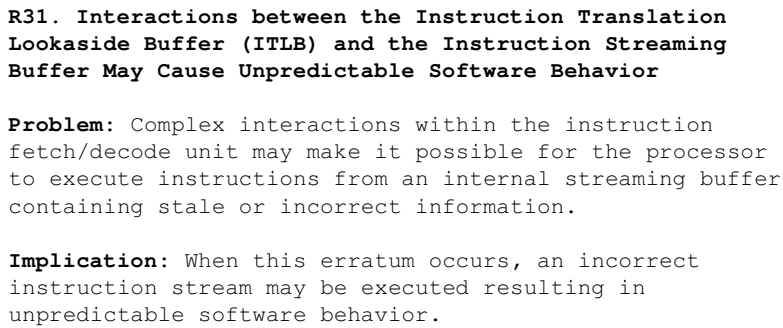

Figure 3. Examples of design bugs from (a) the Pentium 4 errata sheet, and (b) the Opteron errata sheet

mechanism distributes the monitoring and checking process at the flip-flop level.

- We show that our online design bug detection mechanism can be synergistically combined with a previously proposed online hardware defect detection mechanism. The combination implements a thorough solution that provides a high degree of both reliability and dependability to a system operating in the field. We show that the hardware used for the detection of design bugs can partially be used for detecting hardware defects, thereby amortizing the cost of both mechanisms.

\section{Design Bug Analysis}

We first analyze design bugs in a real processor to obtain insights into their characteristics and to develop a mechanism that can flexibly and efficiently detect the occurence of design bugs while the system is in operation.

\subsection{Previous Design Bug Analysis Studies}

The potential of augmenting future microprocessors with online design bug detection has led to a number of studies that analyzed the known design bugs that slipped into recent commercial microprocessors. The objective of these studies was to better understand and gain insights into the characteristics of the known design bugs in existing microprocessors, and extrapolate the expected characteristics of the design bugs of future microprocessors.

Specifically, Avžienis et al. [2] analyzed the known design bugs in the Intel Pentium II since its initial release. More recently, Sarangi et al. [20] analyzed the design bugs in ten modern commercial microprocessors from Intel, AMD, IBM and Motorola, and Narayanasamy et al. [17] analyzed the design bugs in two microprocessors: Intel's Pentium 4 and AMD's Athlon 64. Another study by Wagner et al. [26] analyzed the design bugs in Intel StrongARM SA1100 and IBM PowerPC 750GX. The analysis in all of these studies was based on information extracted from the available microprocessor errata sheets e.g. $[13,1,5]$. An errata sheet is a document published and maintained by the microprocessor manufacturer to provide its customers with details about known microprocessor design bugs. The document provides an assessment of each design bug's severity, the degree to which it can affect the system, a possible set of conditions that can trigger the design bug, any possible workarounds, and sometimes the company's intention to provide a fix in a future version of the product. 


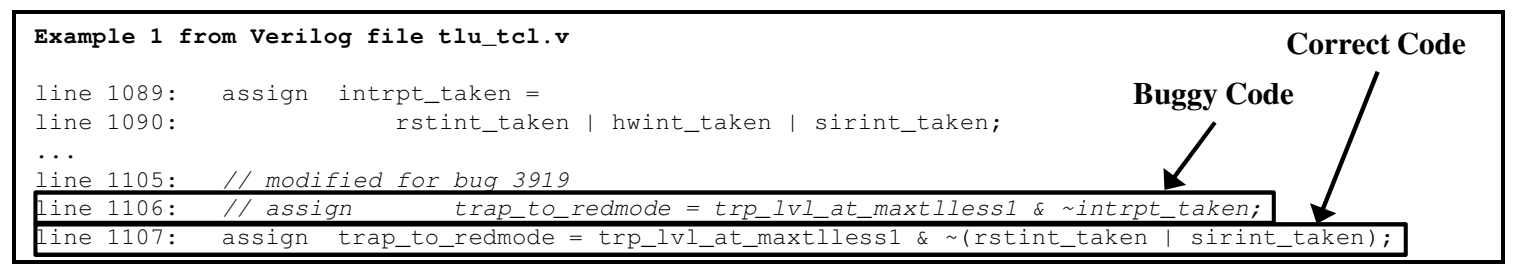

Figure 4. Example of a logic design bug at the RTL level

A major drawback of using the errata sheets to extrapolate statistics about design bugs is that the errata sheets commonly provide very high-level descriptions of the design bugs. Such descriptions provide little or no insight into the low-level details of the underlying hardware problem. An example description of a design bug listed in the Intel Pentium 4 errata sheet [13] is shown in Figure 3(a). This design bug is related to complex interactions between the processor's instruction translation lookaside buffer and the instruction streaming buffer that can result in the execution of an incorrect instruction stream with unpredictable software behavior. Using this description, it is very hard to accurately relate this design bug to the actual hardware implementation and reason about, for example, exactly what hardware signals (i.e., wires) need to be monitored by an online design bug detection mechanism to effectively detect the occurrence of the design bug. Figure 3(b) shows another example design bug description, from AMD's Opteron errata sheet [1]. This bug is related to the translation lookaside buffer flush filter and can lead to unpredictable system behavior. Again, from this high-level description, it is very difficult to infer the set of hardware signals that should be examined to dynamically detect its occurrence. Without knowing the set of hardware signals that needed to be monitored to detect the bug, it is very difficult to design a mechanism that would detect the bug and to accurately estimate the hardware cost of such a mechanism.

Our Goal: In order to design a hardware mechanism that detects design bugs, the signals that affect the occurrence of each bug need to be known. Our goal in this section is to perform a more rigorous, lower-level (RTL) analysis of design bugs. Our purpose is to understand design bug characteristics at the register transfer level to (1) design a flexible mechanism that can detect known design bugs during online operation after the chip is manufactured, and (2) more accurately estimate the hardware cost of such a design bug detection mechanism. To this end, we first draw insights from our analysis of design bugs found and fixed in an existing commercial processor, Sun's OpenSPARC T1.

\subsection{RTL Design Bug Analysis}

We perform an RTL design bug analysis in an attempt to bridge the gap between the high-level design bug descriptions provided by the microprocessor errata sheets and the low-level hardware implementation details needed to devise effective online design bug detection mechanisms. At the RTL level, the microprocessor design behavior is described in a hardware description language (e.g., Verilog or VHDL). This level is considered to be very close to the actual hardware implementation. The only design phases separating the RTL level with the actual hardware implementation are 1) logic synthesis, which generates the design's gate-level netlist and 2) place-and-route, which creates the transistor-level layout of the netlist. Therefore, the direct relation between the RTL level and the underlying implementation provides an adequate level of detail that allows the extraction of low-level design bug characteristics.

Our study focuses on the Verilog RTL source code of the OpenSPARC T1 chip-multiprocessor [23], the open source version of Sun's commercial UltraSPARC T1 (Niagara) chip-multiprocessor. Since no errata documentation is publicly available for the UltraSPARC T1 microprocessor, we focus on the actual design bugs found during the development of the OpenSPARC $\mathrm{T} 1$ and documented in the RTL source code. Specifically, when the designers corrected a design bug, they left the original buggy code in the RTL source file as a comment. Therefore, both the original erroneous implementation as well as the fixed implementation are available in the source code. As such, by examining these two implementations, it is straightforward to discover what hardware signals are involved in each design bug. Although these design bugs did not slip into the final product, we believe they share similar characteristics with the design bugs that eventually slipped into the released version of the microprocessor with the exception of some differences which we discuss in the next section.

Methodology: We analyzed 296 design bugs that were documented in the Verilog source files of two OpenSPARC core units. These bugs account for about $99 \%$ of all documented and commentedout bugs in the OpenSPARC T1 RTL. We classified these bugs into three major classes: 1) Logic design bugs, 2) Algorithmic design bugs, and 3) Timing design bugs. Later, in Section 3, we analyze the logic signals that need to be monitored to detect these bugs.

\subsection{Classification of Design Bugs}

Logic Design Bugs: This class of design bugs is characterized by erroneous logic in combinational circuits. A logic bug occurs because the designer formed an erroneous logic block; for example an AND gate could be used instead of an OR gate, or an inverted signal rather than the non-inverted one. The code segment presented in Figure 4, taken from the OpenSPARC T1 Verilog source files, illustrates an example of a logic design bug. The design bug is located in the core's trap logic unit (TLU) and is associated with the combinational logic that computes the control signal trap_to_redmode. The incorrect combinational circuit implementation is commented out in line 1106. The corrected combinational circuit implementation is shown in line 1107. By examining lines 1089-1090, we notice that the signal replaced in the correct code (intrpt_taken) is computed by ORing three other signals. One of the three signals (hwint_taken) is no longer a source signal in the correct implementation. We observed that many logic design bugs cannot be fixed by simply redefining the logic between the source signals in the buggy implementation. Instead, it is very common that fixing the bug requires the addition or removal of signals to/from the buggy implementation (more than $95 \%$ of logic design bugs had this requirement).

This example demonstrates the amount of low-level information provided in the RTL code that is missing from the design bug descriptions in the errata documentation. For instance, by observing the code segment associated with the design bug, it is very easy to find the set of hardware signals that activate the bug (i.e., trp_lvl_at_maxtllessl, rstint_taken, hwint_taken, and sirint_taken). In analyses solely based on errata sheets, this low-level information is abstracted away in the high-level design bug description and has to be inferred, a process that involves a high amount of uncertainty and inaccuracy.

Algorithmic Design Bugs: This class covers major design bugs related to the algorithmic implementation of the design. These design bugs exhibit algorithmic deviations from the design specification and they usually require major modifications to be fixed. Figure 5 illustrates an example algorithmic design bug located in the load queue control logic at the core's load/store unit. This bug is due to an incorrect implementation of the round robin algorithm for selecting one of 


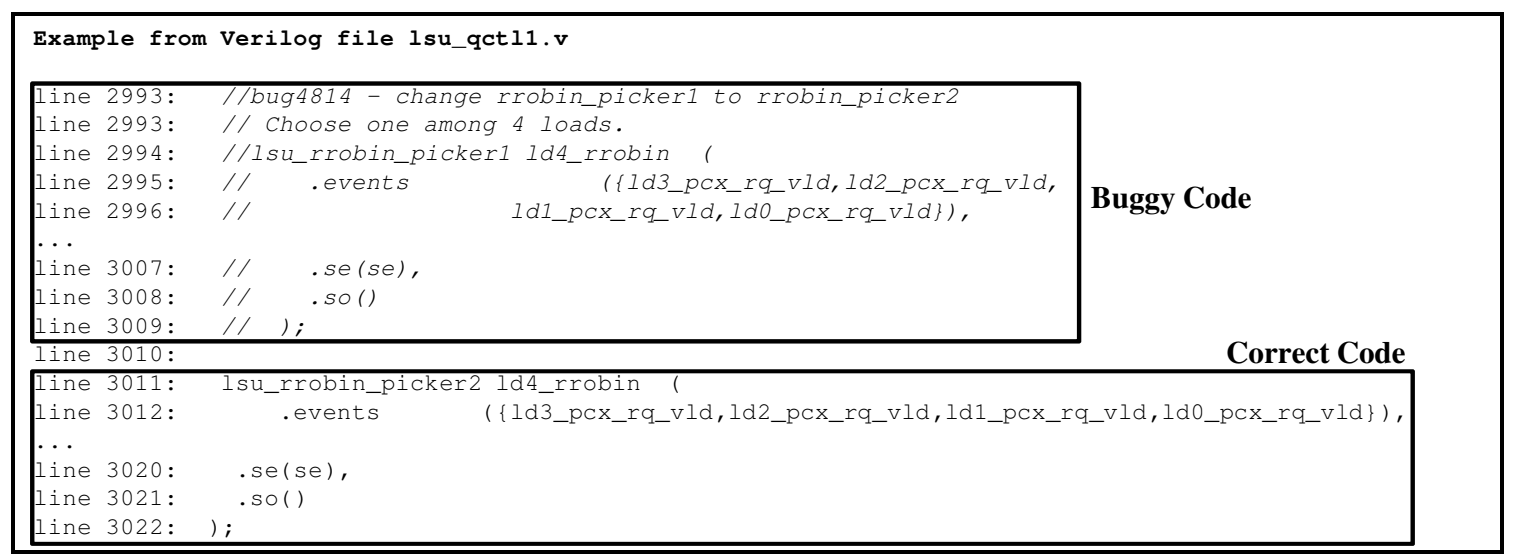

Figure 5. Example of an algorithmic design bug at the RTL level

\begin{tabular}{|c|c|c|}
\hline \multicolumn{3}{|c|}{ Example from Verilog file lsu_qdp1.v } \\
\hline $\begin{array}{l}\text { line 1228: } \\
\text { line } 1239: \\
\text { line } 1240: \\
\text { line } 1241: \\
\text { line } 1242: \\
\text { line } 1243: \\
\text { line } 1244:\end{array}$ & 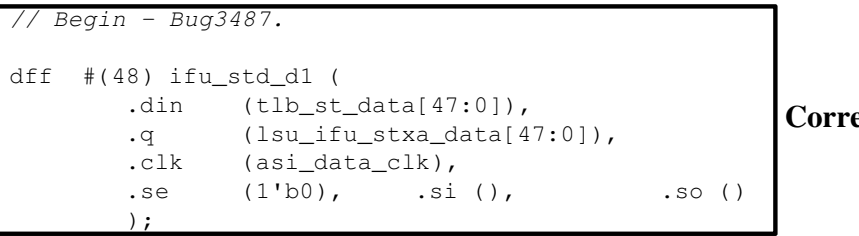 & t Code \\
\hline $\begin{array}{l}\text { line 1245: } \\
\text { line 1246: } \\
\text { line } 1247: \\
\text { line 1248: } \\
\text { line } 1249: \\
\text { line } 1250:\end{array}$ & $\begin{array}{l}\text { T/ select is now a stage earlier, which should be } \\
/ / \text { fine as selects stay constant. } \\
/ / \text { assign lsu_ifu_stxa_data[47:0] }=\text { tlb_st_data_d1[47:0] } \\
/ / \text { End - Bug3487. }\end{array}$ & Buggy Code \\
\hline
\end{tabular}

Figure 6. Example of a timing design bug at the RTL level

the four loads buffered in the load queue. To fix the incorrect round robin implementation described in module lsu_rrobin_picker1, a new module had to be implemented (lsu_rrobin_picker2). Unlike fixes for logic design bugs, fixes for algorithmic design bugs are not limited to combinational circuit modifications, rather they sometimes require multiple major modifications that can span the whole module.

Timing Design Bugs: This third class of design bugs is associated with the timing correctness of the implementation. We have observed that most of these design bugs are cases where a signal needed to be latched a cycle earlier or a cycle later in order to keep the timing of signals correct in the design. An example of such a design bug is shown in Figure 6. This timing design bug is located in the queue data path of the core's load/store unit. As shown in the Verilog source code, the incorrect implementation in line 1248 assigns the value of the 48-bit tlb_st_data_dl bus to the lsu_ifu_stxa_data bus in the same cycle. However, as shown in lines 1239-1244, the correct timing of the data movement between the two buses requires the data to be latched for one clock cycle. We found that the most common fix for this class of design bugs is the addition or removal of flip-flops to adhere to the timing constraints required to keep the design correct.

\subsection{Design Bug Type Distribution}

After studying the OpenSPARC T1 Verilog source files [22] we found that almost all ( $\sim 99 \%)$ of the documented design bugs are located in two units, the load/store unit (LSU) and the trap logic unit (TLU) [23], shown in Figure 7(a). The LSU processes all data memory access instructions. It interfaces with all the functional units and it serves as the gateway between the SPARC core and the core-cache crossbar to the memory subsystem. The LSU also includes the core's data TLB and L1 cache. The TLU implements the SPARC core's trap and software interrupt handling logic. It supports six trap levels ranging from hypervisor and supervisor mode traps to user mode traps and is capable of handling up to 64 pending software interrupts per thread. In our study we analyzed a total of 296 design bugs documented in these two units.

Figure 7(b-c) shows the design bug type distribution. A large fraction of the documented design bugs in the two units belong to the logic design bug class, which accounts for $59 \%$ and $49 \%$ of the total design bugs for the LSU and the TLU, respectively. The second most frequent design bug class is algorithmic design bugs, while timing design bugs are less frequent and account for only $\sim 5 \%$ of all bugs. The dominance of logic design bugs over the other two bug classes might imply that the process of implementing complex combinational logic is more prone to human error than implementing the algorithmic or timing specifications of the design.

As mentioned earlier in this section, these design bugs were discovered, fixed, and documented before the final tape-out of the design. As such, we expect them to have some differences with the design bugs that escape the verification phase and slip into the final product. We suspect that the algorithmic and timing design bugs have a more severe impact on the design's correctness and therefore they might have a higher probability of being discovered during the design verification phase. In contrast, because logic design bugs are isolated and localized to small combinational logic portions, they could be less likely to be discovered during the verification of the chip. This is because the erroneous effects of the logic design bugs either might not be exercised or might be masked before propagating to observable outputs during testing. For example, in order for the logic bug illustrated in Figure 4 to be active, the source combinational circuit must be set to specific values (which might be a rare combination of values). Based on this reasoning, the distribution of design bugs that actually slip into the final product might have fewer algorithmic and timing design bugs than the distribution shown in Figure 7(b-c). 


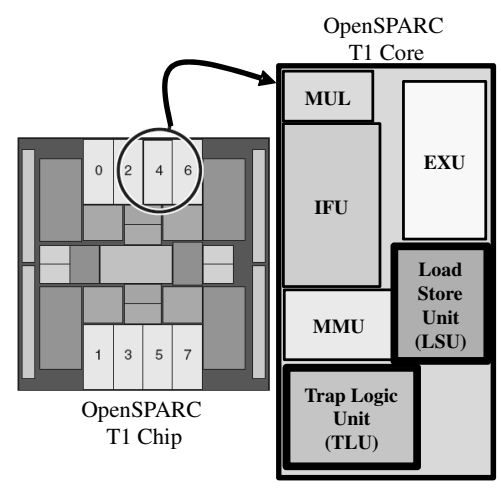

(a) (b)

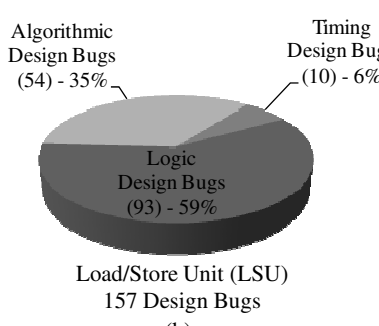

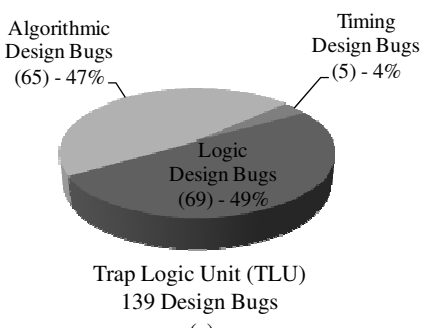

(c)
Figure 7. Part (a) shows the OpenSPARC T1 core. Parts (b) and (c) show the design bug distribution for the Load/Store Unit (LSU) and the Trap Logic Unit (TLU) respectively

\section{Detecting Logic Design Bugs at Runtime}

Although logic design bugs might be harder to discover than the other two design bug classes, we believe that once they have been discovered, it is much easier to detect their occurrence while the "buggy" microprocessor is in operation in the field. Their characteristic of being isolated in a combinational logic circuit portion makes it possible to deterministically detect their occurrence by monitoring the values of their source signals. To illustrate this concept, we consider the logic bug example shown in Figure 4. By computing the truth table of the buggy circuit (line 1106) and the correct circuit (line 1107), as shown in Figure 8(a), we can infer that the design bug occurs when the source signals are set to a specific combination of values (shown in the shaded column of the table). Therefore, by monitoring the values of the bug's source signals it is possible to deterministically detect the occurrence of the specific design bug. In this work, we call this set of signals first-level monitor signals (i.e., signals that directly determine the occurrence of the design bug). For this specific bug, the size of the firstlevel monitor signal set is 4 because there are 4 signals whose values directly determine the bug's occurrence.

Although it is easy to find the set of first-level monitor signals in the RTL model, these signals unfortunately might not exist in the lower transistor-level implementation due to the logic synthesis process and optimizations employed during the process of translating the RTL implementation to gate-level and then to transistor-level implementation [24]. Thus, there is not a guaranteed one-to-one mapping between signals in the RTL and signals in the transistor-level implementations. However, the logic synthesis process maintains a one-to-one mapping of the state-holding elements (e.g., flip-flops) and module-level primary inputs/outputs ${ }^{3}$ between the RTL and transistor-level implementations [24]. To effectively detect the occurrence of a logic design bug in the transistor-level hardware implementation, we need only to trace back the combinational logic that feeds the first-level monitor signals to a set of signals that are directly connected to either 1) state-holding elements or 2) primary inputs of the module. We call this set of signals

${ }^{3}$ In this work we consider a module to be a Verilog design module in the RTL code.

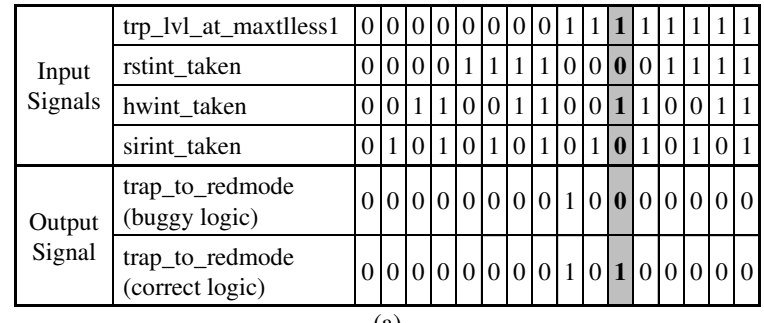

(a)

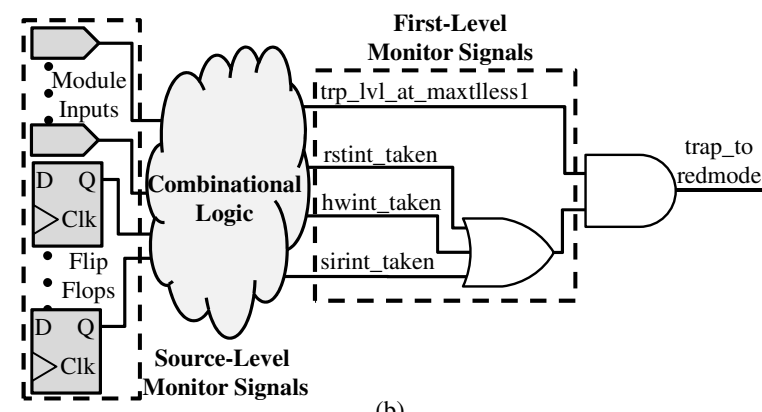

(b)

Figure 8. Part (a): The logic bug shown in Figure 4 is triggered whenever its source signals take the values shown in the shaded column. Part (b): The source-level and first-level signals for the same logic bug

the source-level monitor signals. Figure 8(b) illustrates this process. Monitoring the source-level monitor signal set of a design bug allows the detection of the bug. Note that it is simple to construct a truth table using the source-level monitor signals instead of the first-level monitor signals to understand which combination of the values assigned to source-level signals would exercise the bug.

To determine the number of signals required to be monitored to detect the occurrence of logic design bugs, we measured the first-level and source-level signals of the 162 logic design bugs located in the LSU and the TLU units. Figure 9 shows the cumulative distribution of the logic design bugs versus the first-level and source-level monitor signal set sizes in the LSU and the TLU units. We observe that $97 \%$ of the logic bugs located in the LSU and $92 \%$ of those located in the TLU have a source-level monitor signal set size that is smaller than 64 signals. This means that for detecting any single bug that is within the aforementioned percentage, at most 64 signals need to be monitored.

Table 1 focuses on the number of first-level and source-level signals needed to be monitored to detect logic design bugs. An interesting observation is that the average set size of source-level monitor signals per logic bug is about double the size of the first-level monitor signal set. Notice that the size of the first-level monitor signal set determines the minimum number of RTL signals required to be monitored to precisely detect the occurrence of a certain bug, given that those signals exist in the actual hardware implementation, and can be probed. On the other hand, the size of the source-level monitor signal set determines the number of transistor-level signals required to be tapped to detect a bug, given that design flip-flops and module inputs can be probed. Furthermore, the average number of source-level monitor signals per logic design bug is 17 and 24 for the LSU and the TLU units respectively (The minimum and maximum set sizes are presented as well). Hence, the detection of an average design bug requires 17 to 24 transistor-level signals to be monitored.

The total amount of tapped signals can be small if there is a high degree of source signal sharing between multiple design bugs. To quantify this, we studied the amount of sharing between the 162 logic bugs covered by our study. We found that the sharing between the sourcelevel monitor signal sets is about $65 \%$ on average $(68 \%$ in LSU and $64 \%$ in TLU). This means that $65 \%$ of the signals that belong to the 


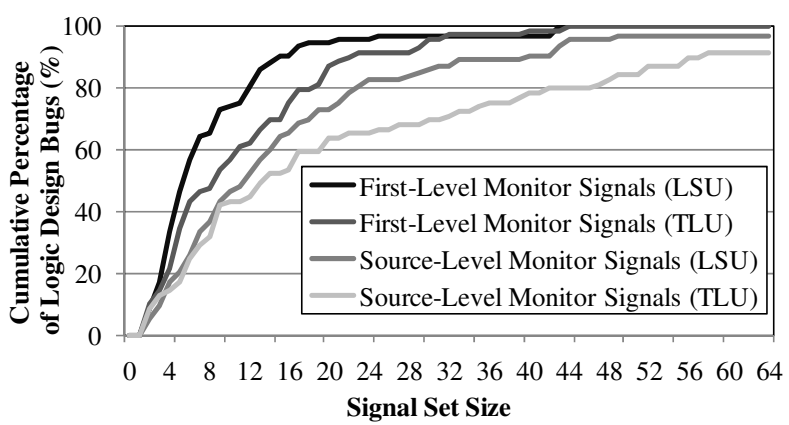

Figure 9. Cumulative distribution of logic bugs versus the first-level and source-level monitor signal set sizes for the LSU and TLU

source-level monitor signal set of a logic design bug also belong to the source-level monitor signal set of at least one other logic design bug. Furthermore, each logic design bug has on average 6-9 signals in its source-level monitor signal set that are unique, i.e., they do not belong to the source-level monitor signal set of any other logic design bug. This result implies that the discovery of a new design bug requires the monitoring of an additional 6-9 signals, on average, that have not been previously monitored for any other bug, thus increasing the total number of tapped signals.

In order to detect all the 162 studied logic design bugs, 516 and 602 unique source-level monitor signals need to be monitored in the LSU and the TLU modules, respectively. Note that these numbers are much higher than previous work estimates that used high-level errata documentation to analyze design bugs. Specifically, the study in [20] reports that on average, for the ten processors studied, only 210 signals need to be monitored to detect all design bugs in all modules of a processor, with the maximum requirement out of the ten microprocessors being 260 signals. The study in [17] reports that monitoring only 41 signals is adequate to detect the occurrence of 43 out of the 63 known design bugs in the AMD Athlon 64 and AMD Opteron microprocessors. In contrast, our study shows that 1118 signals need to be monitored to detect 162 bugs in two modules of the SPARC core. We believe this discrepancy stems from the attempt in previous studies to infer low-level hardware implementation information from the high-level, abstract information provided in the microprocessor errata documents. By studying the documented design bugs at the lower RTL level, we found that the signal monitoring requirements of online design bug detection are significantly higher than the estimates of these previous studies. As a result, the problem of detecting design bugs is more difficult and the solution is likely more hardware intensive than estimated by previous work.

\subsection{Insights from RTL Design Bug Analysis}

In summary, our RTL design bug analysis provides the following conclusions and insights:

1. The design bugs documented in the Verilog source files of the OpenSPARC T1 chip-multiprocessor can be classified into three major classes based on their characteristics: logic, algorithmic, and timing design bugs (Section 2.3).

2. Logic design bugs outnumber the documented design bugs of the other two design bug classes. Furthermore, they might dominate the distribution of design bugs that escape the verification phase and slip into the final product (Section 2.4).

3. Because they only affect combinational logic, the occurrence of logic design bugs is more readily detectable while the system is in operation. This can be done deterministically by monitoring a set of source-level signals.

4. The number of signals that need to be monitored to detect the occurrence of logic design bugs is significantly higher than estimates provided by previous work. The discovery of a new design

\begin{tabular}{|l|c|c|}
\hline \multicolumn{1}{|c|}{ Metrics } & LSU & TLU \\
\hline $\begin{array}{l}\text { Min./Average/Max. number of first-level } \\
\text { monitor signals per logic design bug }\end{array}$ & $2 / 8 / 43$ & $2 / 12 / 44$ \\
\hline $\begin{array}{l}\text { Min./Average/Max. number of source- } \\
\text { level monitor signals per logic design bug }\end{array}$ & $2 / 17 / 97$ & $2 / 24 / 89$ \\
\hline $\begin{array}{l}\text { Source-level monitor signal sharing } \\
\text { among different design bugs }\end{array}$ & $68 \%$ & $64 \%$ \\
\hline $\begin{array}{l}\text { Average number of unique source-level } \\
\text { monitor signals per logic design bug }\end{array}$ & 6 & 9 \\
\hline $\begin{array}{l}\text { Unique source-level monitor signals (for } \\
\text { all logic design bugs) }\end{array}$ & 516 & 602 \\
\hline
\end{tabular}

Table 1. Logic design bug statistics

bug requires the monitoring of additional 6-9 signals, on average, that have not been previously monitored for any other bug.

These conclusions and insights call for a mechanism capable of concurrently monitoring a large number of different signals scattered in the design and thus providing an effective and efficient substrate to perform online detection of the occurrence of logic design bugs. In the rest of the paper we describe our proposal for developing such a mechanism.

\section{Distributed Online Bug Detection}

In this section we present our distributed online design bug detection mechanism. Section 4.1 gives an overview of our mechanism, and Section 4.2 describes how it can be implemented in hardware. Section 4.3 describes how our mechanism is intergrated at the system level.

\subsection{Overview of Online Design Bug Detection}

Figure 10 illustrates the high-level architecture of our online design bug detection mechanism. The mechanism is characterized by two phases: 1) the initial setup of the mechanism and 2) the cycle-by-cycle operation where design bugs are detected while the system is operating in the field.

4.1.1. Initial Setup Process The first step of the mechanism's setup process is the determination of the triggering conditions for each design bug in the system. The design bug triggering conditions are characterized by (1) the bug's source-level monitor signals and (2) their values that would activate the bug. The design bug triggering conditions of each bug are determined by system engineers after performing the bug analysis process presented in Section 3.

Bug Signatures: Once bug triggering conditions are determined, they are represented by a structure called a bug signature (step 1 in Fig. 10). Conceptually, the bug signature is a list of all the signals in the system. From that list, the bug's source-level monitor signals are marked with the value they need to take to trigger the bug, while nonsource signals are marked with a don't care value (X) indicating that their values are irrelevant to the bug activation. The bug signature can be considered as a representation of the system state that would activate the design bug. Each design bug can have multiple bug signatures due to multiple possible combinations of triggering conditions.

System Bug Signature: The next step in setting up the design bug detection mechanism is the generation of the system bug signature. The collection of bug signatures of all design bugs are merged together to form the system bug signature (step 2 in Fig. 10). The system bug signature constitutes a representation of all the conditions that can trigger any individual design bug in the system. The process of merging multiple bug signatures into the system bug signature is detailed in Section 4.2.2.

Bug Detection Segments: The system bug signature is subsequently encoded into a binary representation, partitioned into segments, and loaded into the mechanism's bug detection segments (step 3 


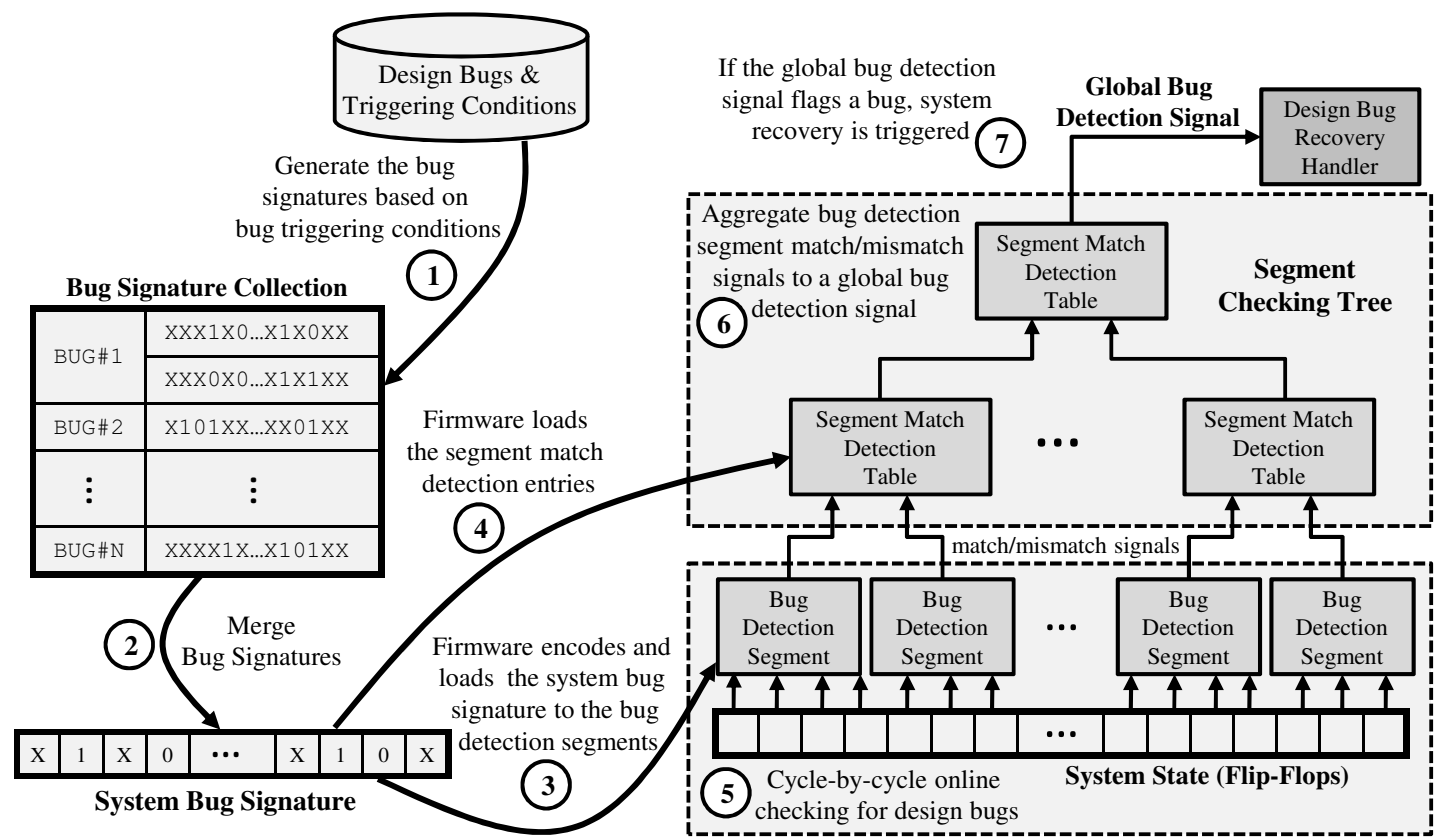

Figure 10. Overview of our online design bug detection mechanism: 1) initial setup (steps 1-4) and 2) online bug detection (steps 5-7)

in Fig. 10). The bug detection segments are field programmable structures each associated with a part of the system state (i.e., the system's flip-flops). Each bug detection segment is loaded with the part of the system bug signature corresponding to its part of the system state. The loading of the bug detection segments is done by firmware that has access to the segments' field programmable resources. During system operation, the bug detection segments compare the system state to the system bug signature and generate match/mismatch signals.

Segment Match Detection Tables: The source-level signals of a design bug might be located only in some of the bug detection segments. Therefore, each bug is associated with a segment match detection entry that indicates which lower-level segments need to match the system bug signature with the system state for the bug to be detected. In essence, the system bug signature summarizes all the triggering conditions from all bugs whereas each segment match detection entry demultiplexes them to enable the detection of individual bugs. The segment match detection entries are loaded into the segment match detection tables by firmware (step 4 in Fig. 10).

\subsubsection{Cycle-by-cycle Operation and Design Bug Detection}

Flip-Flop Level Checking: Once the initial setup of the mechanism is done by the firmware, the remaining task of the mechanism is to check if the system steps into a bug triggering state while it is operating. To check this, each bug detection segment compares its portion of the system bug signature to the system state and generates a segmentwide match/mismatch signal (step 5 in Fig. 10).

Segment Checking Tree: The detection of each individual bug usually requires only a subset of all the bug detection segments in the design to match their portion of system bug signature with the system state. For each bug, this information is encoded into a segment match detection entry. However, the set of segments that are required for the detection of an individual bug might be scattered in different areas of the chip. To aggregate the match/mismatch signals of all the segments on the chip, our mechanism employs a distributed segment checking tree. Each node in the segment checking tree has a segment match detection table that is populated with the segment match detection entries of each bug that has bug-detetcion required segments connected to the tree node. These entries are loaded during the initial setup phase by firmware (step 4 in Fig. 10). During system operation, if the match/mismatch signals of the underlying segments match with one of the node's segment match detection entries, this indicates that the local triggering conditions of a design bug within that node are met. In a similar fashion, each level of nodes in the tree generates a match/mismatch signal and feeds the upper level of nodes (step 6 in Fig. 10). If a match signal propagates all the way from the bug detection segment level to the top level of the tree, this indicates that the triggering conditions of a specific design bug are met for the whole chip and a global bug detection signal is asserted. This process is illustrated in detail with an example in Section 4.2.3. The bug is subsequently flagged to the bug recovery handler (step 7 in Fig 10).

Design Bug Recovery Handler: If a bug is flagged by the global bug detection signal, the design bug recovery handler recovers the system into the last validated system state. Execution is then restarted and guided by design bug avoidance techniques so that the design bug is averted, if possible. Since our focus is on bug detection, we leave the design of the bug recovery handler to future work.

\subsection{Hardware Implementation}

4.2.1. Bug Detection Flip-Flops In our mechanism, the system bug signature and its comparison with system state is distributed to the flip-flop level. This is achieved by augmenting the system flipflops with extra logic for storing the system bug signature and comparing it to the system state. Figure 11(a) shows a system flip-flop augmented with these extensions. The non-shaded logic comprises a scan flip-flop, the common type of flip-flop used in modern processors to enable scan-in and scan-out functionality to facilitate manufacturing testing using Automatic Test Pattern Generation [14, 28]. The system portion is used for holding the system state, while the scan portion is used to scan-in test patterns and scan-out test responses. In current designs, the scan portion is utilized only during the manufacturing testing phase and stays idle during normal operation. During normal operation, our mechanism uses the scan portion in combination with an extra bug detection portion to store the system bug signature. The scan portion is used to indicate whether the specific flip-flop belongs to any bug's source-level monitor signal set. If the scan portion is set to 1 the flip-flop is indicated as a bug source signal, otherwise the flipflop's value is irrelevant to the activation of a design bug. In the former case, the value that will activate the design bug is stored in the bug detection portion. The box at the top of Figure 11(b) illustrates the three encoding rules used to binary map the system bug signature to the bug detection portion (shaded box) and the scan portion (white box). 


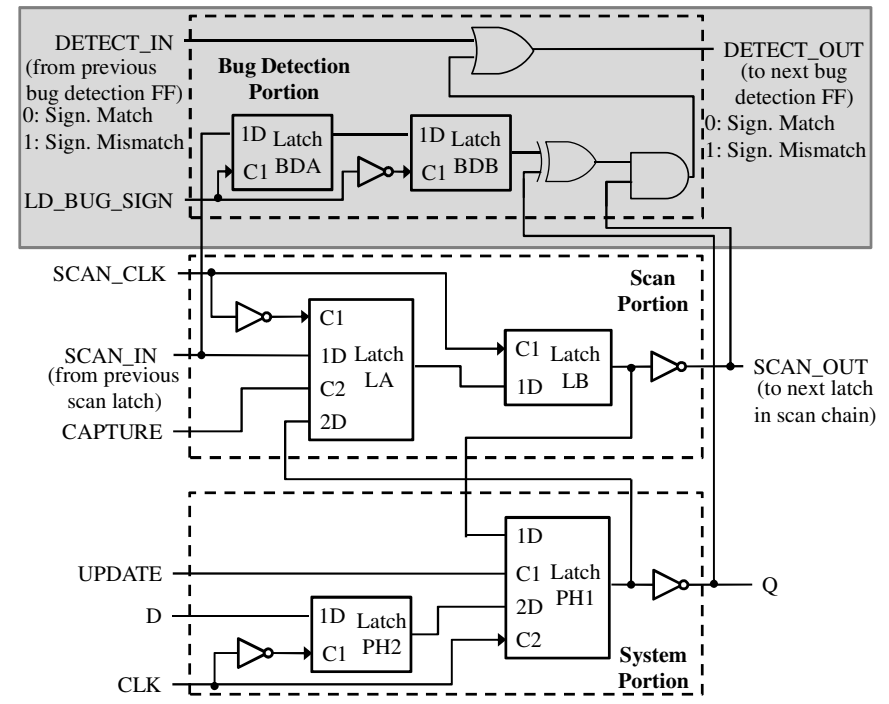

(a)

Figure 11. Modified scan flip-flop with bug detection capabilities

If the scan portion is set, the value of the system flip-flop is compared to the value of the bug detection portion to check if there is a match between the system state and the system bug signature. In our mechanism, flip-flops are grouped into bug detection segments to simplify checking; the comparison result is ORed with the comparison result of the previous flip-flop to generate a segment-wide match/mismatch signal. The signal is propagated to the next flip-flop ( 0 indicates a segment match and 1 indicates a segment mismatch). A bug detection segment consists of multiple bug detection flip-flops connected together in a serial fashion (this is analogous to scan segments in scan chains).

Figure 11(b) demonstrates the system bug signature binary encoding process with an example 8-bit system bug signature. The system bug signature is encoded and loaded into the bug detection and scan portions, and the checking is partitioned into two 4-bit bug detection segments. Figure 11(b) also demonstrates how the bug detection segment signals are generated for two different scenarios. In the first scenario, the system state matches with the system bug signature and the segment bug detection signals are both set to zero indicating that the bug is activated. In the second scenario, the second bit of the system state does not match with that of the system bug signature and therefore the bug detection signal of the particular segment is set to one indicating that the bug is not activated.

4.2.2. Merging Bug Signatures into the System Signature In this section we describe the technique we employ to merge multiple bug signatures to generate the single system bug signature. First, we merge all the bug signatures related to a single design bug into an intermediate bug signature. To do so, for each bit location we check the values of all bug signatures. If the bit takes the value of zero in some signatures and the value of one in others, then a don' $t$ care (X) value is assigned to the merged intermediate bug signature since for that signal either value can lead to a bug triggering combination. If the value of the bit is constant for all signatures then that value is assigned to the merged intermediate bug signature. This technique is illustrated in Figure 12 for two example design bugs.

When merging the intermediate bug signatures of multiple bugs into the system bug signature, we employ a slightly different technique. Again, if a bit location takes both values (one and zero) among different intermediate signatures, it is marked with a don't care. The difference from the previous technique is that now it is possible for a bit location to have a zero or a one in the intermediate signature of one bug and a don't care in the intermediate signa-

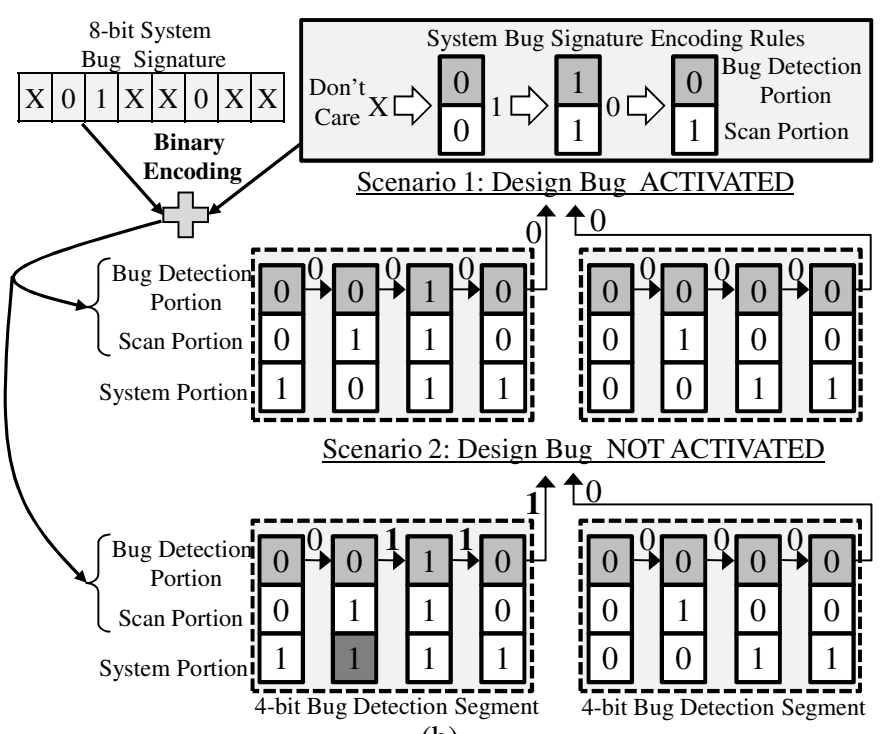

(b)

(a). Example of an 8-bit bug signature encoding and checking (b).

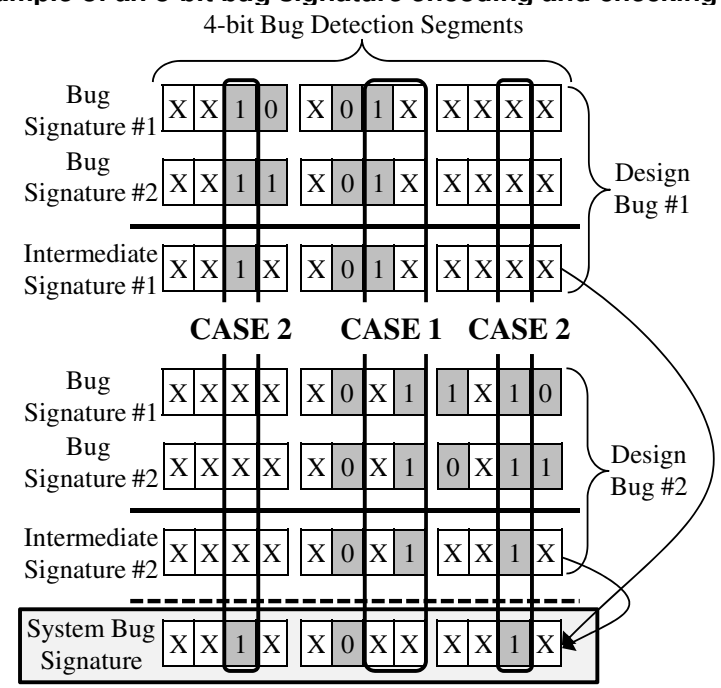

Figure 12. Merging bug signatures into system bug sigmature

ture of another. This case is treated differently depending on the status of the remaining signals in the bug detection segment:

- CASE 1: Consider the two righmost bits of the middle bug detection segment of Figure 12. They both have the value of one in one of the intermediate bug signatures and a don't care value in the other. Since the whole bug detection segment needs to match to trigger a bug and both bugs have other source signals in this bug detection segment (the second source signal with the value zero), the specific source signal is assigned a don't care value so that it will not prevent the detection of any of these two particular design bugs.

- CASE 2: Now, consider the third bits of the leftmost and the rightmost bug detection segments. Again, in one of the intermediate signatures they have the value of one while in the other they have a don't care value. However, in this case no other source signal in the bug detection segments is shared between the two bugs. This means that the segments are associated with only one design bug. Therefore, the source signals can be set to one in the system bug signature because only a single bug requires the particular segment to match its portion of system bug signature with the system state to detect the bug activation.

False Positives - Notice that our mechanism uses don' $t$ care values to merge multiple bug triggering conditions and multiple bug 


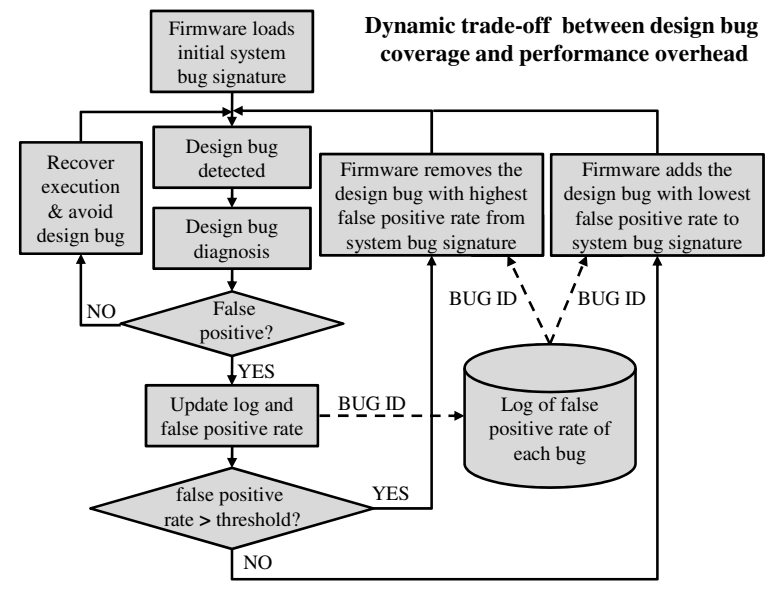

Figure 13. Dynamically changing the set of covered design bugs to regulate the false positive rate and performance overhead

signatures. This approach relaxes the bug triggering conditions and can result in false positives, that is, non-errant conditions which initiate an innocuous recovery sequence. However, since the technique only relaxes the triggering conditions, it cannot exhibit false negatives, that is, discovered design bugs with installed signatures that do not successfully initiate recovery. This is a very important property, since it guarantees that the system will not experience the effects of a specific design bug once the bug is covered by the mechanism.

However, the presence of false positives can adversely impact the performance of the system if too many false recovery alarms are issued. Since the false positive rate highly depends on the dynamic system conditions and workload, we propose a dynamic scheme for trading off design bug coverage with system performance. Figure 13 gives a high-level overview of this scheme. At system start-up, firmware loads into the mechanism the initial system bug signature that covers all design bugs. A triggered design bug detection is followed by a diagnosis process that determines if the design bug detection is correct or if it is a false positive. If the detection is correct, the system execution is recovered and the design bug is averted using design bug avoidance techniques. If the detection is a false positive, then the false positive rate of the specific design bug is logged using the bug's ID tag and the system's false positive rate is calculated. The system's false positive rate is then compared with a predefined threshold. If the system's false positive rate is larger than the threshold, the design bug with the highest false positive rate is removed from the set of covered design bugs and firmware regenerates and loads into the mechanism the new system bug signature. On the other hand, if the system's false positive rate is smaller than the threshold, the design bug with the lowest false positive rate is added to the set of covered design bugs.

The predefined threshold can be adapted dynamically based on the requirements of the running applications. For example, a performance-critical application with low dependability requirements can set the threshold low, while a dependability-critical application can set it high. Furthermore, this scheme can be optimized to achieve the optimum trade-off between design bug coverage and performance overhead due to false positives. Unfortunately, the exploration and evaluation of this technique requires extensive simulation of real workloads on a low-level (RTL) model of the design. To the best of our knowledge, such a simulation infrastructure is not available in our research community. Therefore, we leave this exploration and evaluation for future work.

4.2.3. Segment Checking Tree Implementation In our mechanism, the bug detection segment signals are aggregated to generate one global bug detection signal through a hierarchical tree structure, the segment checking tree. The implementation of this structure is

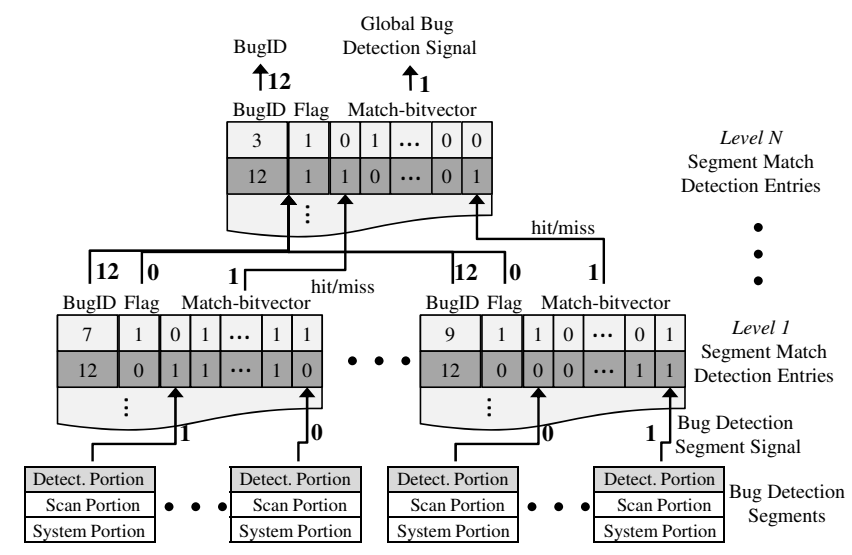

Figure 14. Distributed segment checking tree example

shown in Figure 14. Each leaf node of the structure is connected to a set of bug detection segments. For each bug that has source signals located in bug detection segments assigned to a leaf node, a segment match detection entry is allocated in that node. Each segment match detection entry indicates which subset of the node's bug detection segments need to match the system bug signature to trigger the given bug through the Match-bitvector field. Each entry also has a Bug ID and a Flag field. The Bug ID field indicates the bug associated with the specific entry, while the F lag field indicates whether the specific bug has source signals that are mapped on a different leaf node. For example, the design bug with the ID tag 12, has source signals both in the leftmost and in the rightmost leaf nodes of the tree. Therefore, it is allocated a segment match detection entry in each of those nodes with the Flag field set to zero. On the other hand, the design bugs with ID tags 7 and 9 have source signals limited only to one leaf node and this is indicated by having their Flag field set. A bug that has its Flag field set means that if the Match-bitvector field of that particular bug matches with the values of the underlying bug detection segments, then no further checking is required (since the bug's signals are limited only to that node) and the bug is flagged, along with its ID tag, through the tree to the top level global bug detection signal. Notice that if two bugs are flagged in the same cycle (e.g., bugs 7 and 9 ), only one of them will be flagged to the top level and the decision will be arbitrary based on the implementation. However, due to the rare occurence of design bugs, we don't expect two design bugs to be triggered in the same cycle.

Figure 14 illustrates the detection of the bug with the ID tag 12 . In the specific example, the values generated by the underlying bug detection segments match with the Match-bitvector fields of bug 12 in both leaf nodes. Since the Flag field is set to zero, the bug is not flagged and the hit/miss signal from the leaf nodes are passed to the upper level. When the node hit/miss signals reach the top level node of the tree, the values match with the bug's Match-bitvector entry and therefore the global bug detection signal is set to one, triggering the design bug recovery process, and the bug ID tag is passed to the bug recovery handler.

\subsection{System-Level Integration}

The proposed mechanism requires two additional critical functionalities to provide a complete online bug detection solution:

1. In-the-Field Programmability: The system bug signature and the data that need to be stored in segment match detection entries are dynamic and change as new design bugs are discovered or old bugs get fixed. This part of the design needs to be field-programmable and upgradable by special firmware developed and distributed by microprocessor vendors.

2. Recovery Support: The detection of the occurrence of a design bug is only the first step in providing a solution to the problem. Further action is required to avert the design bug and avoid 


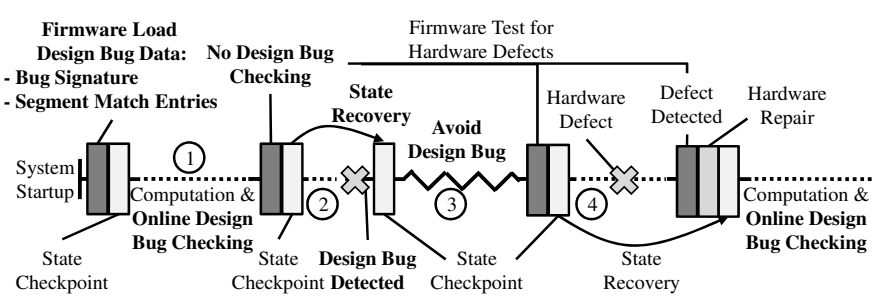

Figure 15. Unifying design bug detection and defect detection

corrupting the execution. This is commonly achieved through recovery support where the system state recovers to the last validated/correct state $[18,21]$ and execution is guided from there in a way that the design bug is averted [19, 26, 17].

In addition, to be widely adopted, a bug detection mechanism needs to have low area and power consumption overhead. To accomplish this, we propose amortizing the cost of the proposed logic by using it for other purposes than solely bug detection (e.g., hardware defect detection).

Unifying Defect Detection and Design Bug Detection - We found that our recently proposed mechanism for detecting hardware defects can provide an efficient substrate for both requirements [4]. This online defect detection technique introduces the Access/Control Extension (ACE) framework to provide firmware access to the processor's scan state. This functionality is used to load ATPG test patterns and test the underlying hardware through specialized firmware. The ACE framework also uses a tree structure to access the scan state, similar to the tree structure used in this work to maintain the segment match detection entries and perform distributed bug checking (see Figure 14). Since the ACE framework can read/write to any of the tree nodes and any scan flip-flop in the design, it can also be used to program the segment match detection entries in the distributed bug checking tree and load the bug signature at the flip-flop level. We believe that this framework can be easily adapted to provide the bug detection mechanism programmability through firmware with minor engineering changes.

Checkpointing \& Recovery: The same framework also employs coarse-grained checkpoint and recovery techniques, based on previous work $[18,21]$, to provide recovery from hardware defects. We believe these checkpoint and recovery techniques can offer an effective substrate to provide recovery support to our mechanism. By rolling back the system state to the last validated and correct system state, execution can be guided by design bug avoidance techniques in a way to avert the design bug. Several design bug avoidance techniques have already been proposed in the research literature [19, 26, 17]. Any further advancement toward this direction is left for future work.

System-Level Operation: Furthermore, the two mechanisms can work together synergistically and provide a collective solution for reliable and dependable computing. Figure 15 shows the synergistic execution timeline of the two mechanisms. At system startup, special firmware uses the ACE framework to load the bug signature and the segment match detection entries needed for online design bug detection. During a checkpoint interval, execution is guarded from the effects of design bugs by our online bug detection design (phase 1). If no design bug is detected, at the end of the checkpoint interval special firmware uses the ACE framework to test the underlying hardware for defects as described in [4]. If the test succeeds, a new checkpoint is taken. If, during the checkpoint interval, a design bug is detected, the system state is rolled back to the last checkpoint (phase 2) and bug avoidance techniques are employed to avoid the design bug (phase 3 ). If a hardware defect manifests during a checkpoint (phase 4), the defect is detected at the end of the checkpoint and, after system repair, the system state is rolled back to the last checkpoint for re-execution as described in [4]. Notice that the use of the tree resources and the scan state is mutually exclusive by the two mechanisms. The online design bug detection mechanism utilizes these resources during a checkpoint

\begin{tabular}{|l|l|}
\hline Methodology/Tools Used & \multicolumn{1}{c|}{ Design Components } \\
\hline \multirow{5}{*}{ Synopsys Power Compiler } & $\begin{array}{l}\text { 1) SPARC Cores, 2) Crossbar, 3) FPU, } \\
\text { 4) Misc. Units (I/O Bridge, DRAM } \\
\text { Controllers, Control \& Test Unit) } \\
\text { 5) ACE Framework, 6) Online Design } \\
\text { Bug Detection Mechanism }\end{array}$ \\
\hline CACTI 4.2 & 1) L1 Inst. \& Data Caches, 2) L2 Cache \\
\hline Taken from [15] & 1) I/O Pads, 2) Wires \& Repeaters \\
\hline
\end{tabular}

Table 2. Power consumption estimation methodology

interval, while the hardware defect detection mechanism utilizes the resources at the end of a checkpoint interval. Hence, the cost of the tree is amortized between bug detection and defect detection.

\section{Experimental Evaluation}

\subsection{Experimental Methodology}

The case study design used for the experimental evaluation of our mechanism is the OpenSPARC T1 chip-multiprocessor, the opensource version of Sun's Niagara (UltraSPARC T1) [22]. We choose this design because the OpenSPARC T1 chip-multiprocessor targets commercial applications such as database and web servers where system correctness is of paramount importance. We believe such systems constitute ideal candidates to employ our mechanism to provide the required correctness guarantees. The OpenSPARC T1 is a full-system multiprocessor design implementing the 64-bit SPARC V9 architecture. It contains eight 6 -stage pipelined in-order cores, each with $8 \mathrm{~KB}$ L1 data-cache, 16KB L1 instruction-cache and full hardware support for four threads. The eight cores are connected through a crossbar to a unified 3MB L2 cache and a shared floating-point unit. The chip also includes four memory controllers and an input/output bridge [23].

RTL Implementation: To make an accurate assessment of our mechanism's requirements in silicon area and power consumption, we developed a detailed RTL model of our mechanism in Verilog. Specifically, in our prototype we implemented 1) the bug detection flip-flops that hold the bug signature and compare it with the system state, 2) the segment checking tree with a parameterized number of segment match detection entries per tree node, and 3) the ACE-based field programmable framework that loads through firmware the bug signature and the segment match detection entries. Our implementation covers all modules in OpenSPARC T1 except the memory cache data and tag arrays (we don't expect logic design bugs to be located in regular and meticulously optimized arrays).

Logic Synthesis and Tools: We used the Synopsys Design Compiler to perform logic synthesis on the RTL code of the OpenSPARC T1 and our mechanism. Logic synthesis mapping is done using the Artisan IBM 0.13um standard cell library. The resulting gate-level netlists of the OpenSPARC design and our mechanism provided a common substrate to accurately estimate the silicon area and power consumption overhead on the whole OpenSPARC design.

Power Consumption Estimation Methodology: To evaluate the power consumption overhead of our mechanism, we first estimated the power consumption of the baseline OpenSPARC T1 design without the extra hardware required by our mechanism. We calibrated the estimated power consumption with actual power consumption numbers provided by Sun for each module of the chip [15]. After we validated our power estimates for the baseline OpenSPARC T1 design, we estimated the additional power required by our mechanism. Table 2 shows the major design components of the OpenSPARC T1 and the methodology/tools we used to estimate their power consumption. We estimated the power consumption of the majority of the OpenSPARC T1 modules using the Synopsys Power Compiler (part of the Synopsys Design Compiler package). To estimate the power consumption of the L1 and L2 caches, we used the CACTI 4.2 tool [25], a tool with integrated performance, area, and power models for memory cache structures. 


\begin{tabular}{|l|c|c|}
\hline \multicolumn{1}{|c|}{ Chip Submodule } & Data Signals & Control Signals \\
\hline SPARC Core $(x 8)$ & $15632(79.06 \%)$ & $4140(20.94 \%)$ \\
\hline CPU-Cache Crossbar & $27283(98.69 \%)$ & $362(1.31 \%)$ \\
\hline Floating-Point Unit & $4054(87.75 \%)$ & $566(12.25 \%)$ \\
\hline Control \& Test Unit & $2325(55.29 \%)$ & $1880(44.71 \%)$ \\
\hline Input/Output Bridge & $10251(95.14 \%)$ & $524(4.86 \%)$ \\
\hline DRAM Controller $(\mathrm{x} 4)$ & $13449(94.70 \%)$ & $752(5.30 \%)$ \\
\hline Total & $222765(84.95 \%)$ & $39460(15.05 \%)$ \\
\hline
\end{tabular}

Table 3. Fraction of data and control signals in the OpenSPARC T1

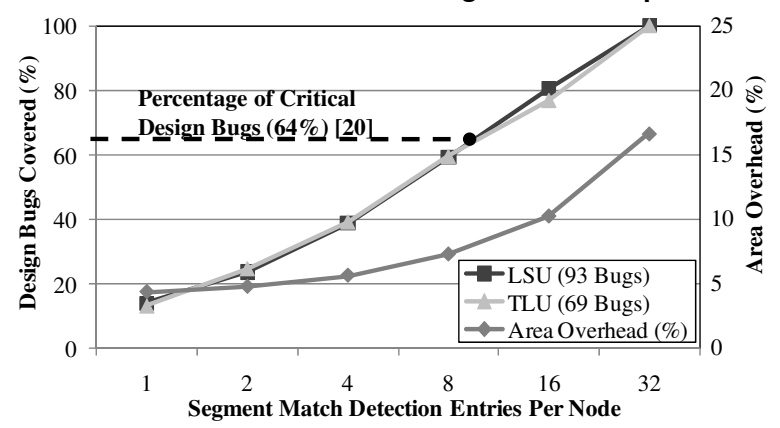

Figure 16. Area overhead versus design bug coverage

This methodology is sufficient to estimate the power consumption of most of the chip's logic modules. However, there are parts of the design whose power consumption cannot be accurately estimated with these tools. These include 1) numerous buses, wires, and repeaters distributed all over the design, which are very hard to model accurately using the Power Compiler, unless the design is fully placed and routed, 2) I/O pads of the chip. In order to estimate the power consumption of these two parts, we used values from the reported power envelope of the commercial Sun UltraSPARC T1 design [15].

\subsection{Area Overhead and Design Bug Coverage}

Control vs. Data Signals - After synthesizing the OpenSPARC T1 chip we found that there are about $262 \mathrm{~K}$ flip-flops in the design. We also found that providing monitoring and bug detection capabilities for all these signals results in prohibitive area overhead $(\sim 69 \%)$. However, we observed that the majority of these flip-flops serve as buffers to data busses or data registers, and only a small fraction of them are control signals. Furthermore, after analyzing the source signals of the logic design bugs studied in Section 3, we found that all of the bug source signals were control signals, and no logic design bug had a source signal that was part of a data bus or a data register. After this observation, we partitioned the flip-flops of the OpenSPARC $\mathrm{T} 1$ design into data and control signals. Table 3 shows the fraction of data and control signals for all modules in the OpenSPARC T1. For the whole chip, only $39 \mathrm{~K}$ flip-flops drive control signals, accounting for $15 \%$ of all flip-flops in the design.

Our prototype implementation taps all $39 \mathrm{~K}$ control signals in the OpenSPARC T1 design. This means that each of these flip-flops is augmented with the extra bug detection logic shown in Figure 11(a). The area overhead of this flip-flop augmentation is estimated to be $3 \%$. Flip-flops are grouped into 8-bit bug detection segments and connected to a four-level segment checking tree structure (shown in Figure 14). The area overhead of the tree structure depends on the number of segment match detection entries per tree node. The number of design bugs that can be covered by our mechanism also depends on the number of segment match detection entries per tree node, raising an engineering trade-off between area overhead and bug coverage.

Area Overhead vs. Coverage - Figure 16 illustrates this trade-off based on the 162 logic design bugs located in the SPARC core's LSU and TLU units studied in Section 3. The figure depicts the percentage of design bugs covered (left Y-axis) and the area overhead (right

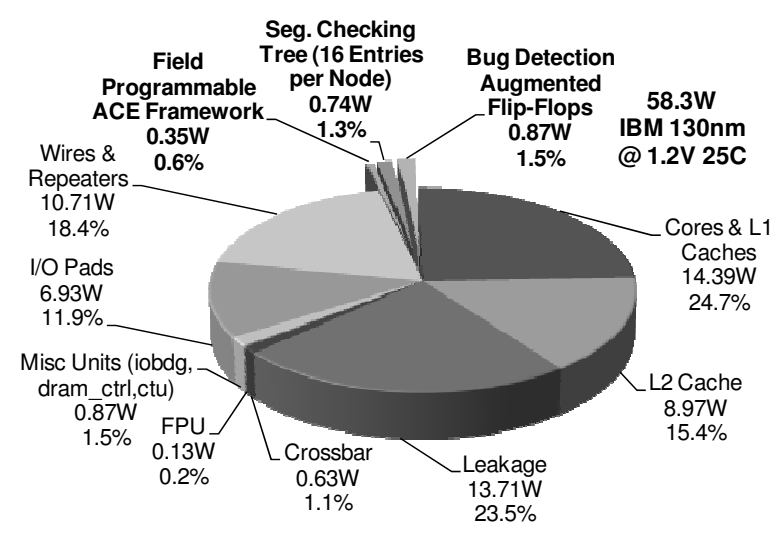

Figure 17. Power consumption overhead

Y-axis) versus the number of segment match detection entries per tree node. When the tree nodes are equipped with 32 entries, our mechanism can cover all the 162 design bugs with an overall area overhead of $17 \%$. Fortunately, not all design bugs are critical to functional correctness and need to be covered. Sarangi et al. [20] studied the errata documentation of ten modern microprocessors and found that, on average for all the studied processors, $64 \%$ of the design bugs are critical to functional correctness. The remaining $36 \%$ of the design bugs were found to be non-critical to the correctness of the system and commonly located in modules such as performance counters, error reporting registers, or breakpoint support [20]. In Figure 16, we can observe that 16 segment match detection entries per tree node provide a design bug coverage of $80 \%$ that is much higher than the typical fraction of critical design bugs. This design configuration leads to a silicon area overhead of $10 \%$ of the whole OpenSPARC T1 design.

\subsection{Power Consumption Overhead}

Employing the methodology described in Section 5.1, we estimated the power envelope of the baseline OpenSPARC T1 chip, without the additional hardware required by our mechanism, to be $56.3 \mathrm{~W}$. Our estimate of the OpenSPARC T1 power is within $12 \%$ of the reported power consumption of the commercial Sun Niagara design [15]. Figure 17 shows the power consumption for our enhanced OpenSPARC T1 design including our online design bug detection mechanism. The power envelope of the enhanced design is $58.3 \mathrm{~W}$. From this, a total of $3.4 \%(1.96 \mathrm{~W})$ is devoted to the extra hardware required by our mechanism. The overall power consumption overhead of our mechanism over the baseline is therefore about $3.5 \%$.

\subsection{Unified Design Bug \& Defect Detection Overhead}

As illustrated in Section 4.3, our online design bug detection mechanism can be easily coupled with an online ACE-based hardware defect detection mechanism. Table 4 presents the silicon area and power consumption overhead of the unified mechanism. The estimated silicon area overhead of the unified mechanism is $15.15 \%$, and its power consumption overhead is $6.8 \%$. Based on these numbers, we believe that the coupling of the two mechanisms provides an attractive and relatively low overhead solution for high-dependability computing.

\section{Comparison to Related Work}

Design Bug Analyses: Our online design bug detection mechanism is based on insights from this work and previous design bug analyses that characterize the known design bugs of existing processors. Section 2.1 provides a discussion on previous design bug analyses and how our RTL design bug analysis differs from those previous works.

Online Design Bug Detection: Recently, studies have brought to attention the increasing rate of discovered design bugs in modern processors $[20,17,26]$. These works suggest employing in-the-field design bug detection and recovery as an approach to mitigate the negative 


\begin{tabular}{|l|c|c|c|}
\hline \multicolumn{1}{|c|}{ Mechanism } & $\begin{array}{c}\text { Flip-Flops } \\
\text { Covered }\end{array}$ & $\begin{array}{c}\text { Area } \\
\text { Overhead }\end{array}$ & $\begin{array}{c}\text { Power } \\
\text { Overhead }\end{array}$ \\
\hline $\begin{array}{l}\text { Online Design } \\
\text { Bug Detection } \\
\text { (16 seg. comparator } \\
\text { entries per tree node) }\end{array}$ & 39K Flip-Flops & $10.26 \%$ & $3.5 \%$ \\
\hline $\begin{array}{l}\text { Online Hardware } \\
\text { Defect Detection }\end{array}$ & 262K Flip-Flops & $5.8 \%$ & $4 \%$ \\
\hline $\begin{array}{l}\text { Online Design } \\
\text { Bug Detection } \\
+\end{array}$ & $\begin{array}{c}\text { 39K Flip-Flops } \\
\text { (bug detection) }\end{array}$ & $15.15 \%$ & $6.8 \%$ \\
$\begin{array}{c}\text { Online Hardware } \\
\text { Defect Detection }\end{array}$ & $\begin{array}{c}\text { 262K Flip-Flops } \\
\text { (defect detection) }\end{array}$ & & \\
\hline
\end{tabular}

Table 4. Cost of combined bug and defect detection

effects of design bugs. As with our mechanism, online design bug detection is facilitated by a signal monitoring substrate. Our work differs from these previous works in the following ways:

1. Higher Flexibility: In all these works, the signal monitoring substrate is limited to a small set of signals selected at design time when the design bugs are still unknown. This constitutes a major limitation of these previously proposed mechanisms. Specifically, if a design bug is discovered after product release and its bug triggering conditions involve signals not included in the set of signals selected to be monitored by the substrate, the occurrence of the design bug cannot be detected effectively by those mechanisms. This leads to greatly inflexible design bug detection mechanisms whose effectiveness depends on decisions made at design time based on assumptions regarding the set of signals that would be involved in unknown design bug triggering conditions. Our online design bug detection mechanism addresses this limitation with a novel field-programmable substrate capable of monitoring all control signals in the design that can trigger a design bug. This capability waives the requirement of selecting the set of signals to be monitored at design time and allows this decision to be made after product release when the design bugs and their triggering conditions are known.

2. Synergy with Other Mechanisms: We show that our mechanism can synergistically share its resources with other mechanisms to amortize cost and provide a complete highdependability solution. In contrast, the mechanisms proposed in previous works are specialized for only design bug detection.

Online Hardware Defect Detection: Our mechanism relies on a combination of field-programmable flip-flops and a fieldprogrammable tree structure to provide flexible and efficient in-thefield signal monitoring and bug detection. This support is similar to the support needed for flexible online defect detection techniques [4]. As we showed in Section 4.3, defect detection and bug detection can be combined seamlessly and the hardware cost of the tree structure can be amortized.

\section{Conclusions}

This paper provided a rigorous analysis of processor design bugs in the RTL code of a commercial microprocessor, Sun's OpenSPARC T1 chip. Our low-level analysis of design bugs concluded that the signal monitoring requirements of online design bug detection are significantly higher than the estimates of previous studies. We believe that this discrepancy stems from the attempt in previous studies to infer low-level hardware implementation information from the high-level, abstract information provided in the microprocessor errata documents.

Based on the insights obtained from our rigorous design bug analysis, this paper proposed a novel distributed online bug detection mechanism. The proposed mechanism is able to flexibly monitor all control signals. This approach enables flexibility in bug detection because, unlike previous proposals, it does not rely on the successful selection of relevant signals at design time. Instead, any signal that can participate in the exercising of a bug can be monitored as needed.
We evaluated the cost of our mechanism based on a detailed RTL prototype implementation. The silicon area overhead incurred by our mechanism is $10 \%$ of the whole OpenSPARC T1 chip, while the power consumption overhead is only $3.5 \%$. We showed that the hardware cost of the proposed technique can be somewhat amortized by combining it with a previously proposed online hardware defect detection mechanism that relies on similar field-programmable resources.

We believe that the processor design bug analyses presented in this paper provide an important step in the understanding of design bugs and the requirements to detect and tolerate them. The proposed online design bug detection mechanism is the first fruit of that understanding. We hope the analyses and frameworks provided in this paper will lead to other, similar techniques that will enable the building of systems with high levels of dependability during operation.

Acknowledgments: This work was supported by grants from NSF, SRC, and GSRC.

\section{References}

[1] Advanced Micro Devices. Revision Guide for AMD Athlon 64 and AMD Opteron Processors, Pub. No. 25759 Rev. 3.75, Feb. 2008.

[2] A. Avžienis and Y. He. Microprocessor entomology: A taxonomy of design faults in COTS microprocessors. In DCCA-99, pages 3-24, 1999.

[3] F. Bacchini, R. Damiano, B. Bentley, K. Baty, K. Normoyle, M. Ishii, and E. Yogev. Verification: what works and what doesn't. In DAC-41, 2004.

[4] K. Constantinides, O. Mutlu, T. Austin, and V. Bertacco. Software-based online detection of hardware defects: Mechanisms, architectural support, and evaluation. In MICRO-40, 2007.

[5] Freescale Semiconductor. MPC7457 Chip Errata, Rev. 10, Nov. 2004.

[6] A. Gluska. Coverage-oriented verification of Banias. In DAC, 2003.

[7] R. Goering. Post-silicon debugging worth a second look. In EETimes, Feb. 052007.

[8] Intel Corporation. Intel Xeon Processor - Specification Update, Doc. No. 249678056, Dec. 2006.

[9] Intel Corporation. Intel Core 2 Extreme Processor X6800 and Intel Core 2 Duo Desktop Processor E6000 and E4000 - Specification Update, Doc. No. 313279-024, Feb. 2008.

[10] Intel Corporation. Intel Core Duo Processor and Intel Core Solo Processor on 65nm Process - Specification Update, Doc. No. 309222-016, Feb. 2008.

[11] Intel Corporation. Intel Pentium M Processor - Specification Update, Doc. No. 252665-033, Jan. 2008.

[12] Intel Corporation. Intel Pentium 4 Processor - Specification Update, Doc. No. 249199-069, May 2007.

[13] Intel Corporation. Intel Pentium 4 Processor on 90nm Process - Specification Update, Doc. No. 302352-031, Sept. 2006.

[14] R. Kuppuswamy, P. DesRosier, D. Feltham, R. Sheikh, and P. Thadikaran. Full hold-scan systems in microprocessors: Cost/benefit analysis. ITJ, 8(1), Feb. 2004.

[15] A. S. Leon, K. W. Tam, J. L. Shin, D. Weisner, and F. Schumacher. A power-efficient high-throughput 32-thread SPARC processor. IEEE Journal of Solid-State Circuits, 42(1), 2006.

[16] M. Magee. Intel's hidden Xeon, Pentium 4 bugs. http://www.theinquirer.net, Aug. 2002.

[17] S. Narayanasamy, B. Carneal, and B. Calder. Patching processor design errors. In ICCD, Oct. 2006.

[18] M. Prvulovic, Z. Zhang, and J. Torrellas. ReVive: Cost-effective architectural support for rollback recovery in shared-memory multiprocessors. In ISCA-29, 2002.

[19] S. Sarangi, S. Narayanasamy, B. Carneal, A. Tiwari, B. Calder, and J. Torrellas. Patching processor design errors with programmable hardware. IEEE Micro, 2007.

[20] S. R. Sarangi, A. Tiwari, and J. Torrellas. Phoenix: Detecting and recovering from permanent processor design bugs with programmable hardware. In MICRO, 2006.

[21] D. J. Sorin, M. M. K. Martin, M. D. Hill, and D. A. Wood. SafetyNet: Improving the availability of shared memory multiprocessors with global checkpoint/recovery. In ISCA, 2002.

[22] Sun Microsystems Inc. OpenSPARC T1. http://opensparc-t1.sunsource.net/.

[23] Sun Microsystems Inc. OpenSPARC T1 Microarchitecture Specification. 2006.

[24] Synopsys. Design Compiler User Guide, Version 2002.05, Jun. 2002.

[25] D. Tarjan, S. Thoziyoor, and N. P. Jouppi. Cacti 4.0. Technical report hpl-2006-86, Hewlett-Packard, 2006.

[26] I. Wagner, V. Bertacco, and T. Austin. Using field-repairable control logic to correct design errors in microprocessors. IEEE TCAD, 27(2):380-393, Feb 2008.

[27] A. Wolfe. For Intel, it's a case of FPU all over again. In EE Times, May 1997.

[28] T. Wood. The test and debug features of the AMD-K7 microprocessor. In ITC, 1999. 